Proceedings of the 17th Czech and Slovak Conference on Magnetism, Košice, Slovakia, June 3-7, 2019

\title{
Structural and Magnetic Properties of Mechanically Alloyed $\mathrm{Fe}_{50} \mathrm{Co}_{50}$ Systems
}

\author{
K. Brzózka ${ }^{a}$, T. Szumiata ${ }^{a}$, D. Olekšáková ${ }^{b}$ And P. KolláR ${ }^{c}$ \\ ${ }^{a}$ University of Technology and Humanities, Faculty of Mechanical Engineering, \\ Department of Physics, 26-600 Radom, E. Stasieckiego 54, Poland \\ ${ }^{b}$ Institute of Manufacturing Management, Faculty of Manufacturing Technologies, \\ Technical University of Košice, Bayerova 1, 08001 Prešov, Slovakia \\ ${ }^{c}$ Institute of Physics, Faculty of Science, P.J. Šafárik University, \\ Park Angelinum 9, 04154 Košice, Slovakia
}

\begin{abstract}
The nanostructured Fe-Co alloys were prepared by mechanical alloying of elemental powders using a highenergy ball mill. This made it possible to study the structural evolution of the milling product with the grinding time. In addition to previous X-ray diffraction and thermomagnetic investigations, ${ }^{57} \mathrm{Fe}$ based Mössbauer transmission spectroscopy was used for this purpose. Iron was alloyed with cobalt just after one hour of milling and bcc $\mathrm{Fe}-\mathrm{Co}$ solid solution was formed. The analysis of the hyperfine magnetic field distribution proved that no significant structural changes occurred during further milling. A slight but regular increase in the standard deviation was observed, indicating an enhancement of topological disorder or/and an increase in internal stresses.
\end{abstract}

DOI: 10.12693/APhysPolA.137.726

PACS/topics: mechanical alloying, Fe-Co alloys, nanostructured magnetic materials

\section{Introduction}

Mechanical alloying is one of easily-operated and effective methods to obtain nanostructured alloys with a given composition, including metal-metal systems $[1,2]$. This proceeds due to many processes occurring at the microscopic level, such as repeated cold welding, fracturing, mixing and re-welding of particles used as a substrate, resulting in size reduction and sometimes in chemical reactions. One of the main features of the product in the form of nanostructured powder is a large fraction of grain boundaries in comparison to grain interior, as well as structural differences between them [3-5]. In addition, the internal strain and the number of crystal lattice defects increase with decreasing grain size obtained by long-term grinding. All these features modify the properties of the final powder compared to the bulk material of similar chemical composition.

Among others, iron-cobalt nanocrystalline alloys can be produced in this way. It was reported [6-10] that mechanically alloyed $\mathrm{Fe}_{100-x} \mathrm{Co}_{x}$ systems show the bcc structure for cobalt contribution from $x=0$ up to 70 , while for higher cobalt content the fcc structure also occurs [6]. In the both cases, a solid solution structure with different degrees of disorder was obtained. Just like in corresponding coarse-grained alloys for some cobalt concentration $(40 \leq x \leq 60)$ the ordering was observed consisting in transformation from bcc structure to $B 2$ below the temperature of $720^{\circ} \mathrm{C}$ after cooling $[12,13]$.

corresponding author
Fe-Co alloys are known to have many applications in a wide variety of fields due to an unique combination of their excellent soft magnetic properties: high Curie temperatures, high saturation magnetization, high permeability, relatively high magnetostriction, and low magnetocrystalline anisotropy with good strength [9-14]. All the characteristics depend not only on proportion between the elements, but also on the powder structure. For example, in $\mathrm{Fe}-\mathrm{Co}$ systems, different evolution of saturation magnetization with changing cobalt concentration was observed. In as-milled material, minimum of saturation magnetization for $x=40-50$ is reported [13], and for thermally treated powders, maximum at about $x=30$ is observed, related to the increasing order parameter [15].

The Mössbauer spectroscopy, as a microscopic and very sensitive to the immediate surrounding of the Mössbauer probe method, can provide important information on the microstructure of the powders tested. In this paper it was therefore used to investigate the effect of grinding time on structural evolution and properties of the milling product.

\section{Experimental details}

Mixtures of $\mathrm{Fe}$ and $\mathrm{Co}$ elemental powders in ratio 50:50 were milled in a high-energy planetary ball mill (RETSCH PM4000) with hardened steel balls and vials and ball-to-powder ratio of 15:1, under a protective argon atmosphere. The milling time $t$ was: $1,5,10,15$, 20 , and $40 \mathrm{~h}$. In addition to previous X-ray diffraction and thermomagnetic investigations [13], ${ }^{57} \mathrm{Fe}$ based Mössbauer transmission spectroscopy was used for this 
purpose. Room temperature Mössbauer spectra were recorded using a ${ }^{57} \mathrm{Co} / \mathrm{Rh}$ source of radiation and a conventional spectrometer working in a constant acceleration mode, with a resolution of $2 \times 512$ channels. Spectra were fitted by means of program NORMOS using the Hesse-Rübartsch method [16] to derive a histogramlike distribution of hyperfine magnetic field (HMF) correlated with isomer shift distribution. Isomer shift values are quoted in relation to the metallic iron. An attempt was also made to use a discrete HMF distribution based on the local environment model.

\section{Results and discussion}

Previous XRD investigations performed for $\mathrm{Fe}_{100-x} \mathrm{Co}_{x}$ systems [13] showed that after $30 \mathrm{~h}$ of milling the alloys with the structure of bcc solid solution were formed, which was also confirmed by the course of thermomagnetic curves. Average grain diameter derived for $x=50$ was in the range $8.5-15 \mathrm{~nm}$, and the internal micro-strain was within the limits $(0.4-0.6) \%$. The magnetic characteristics as coercivity and saturation magnetization of the milled alloys strongly depended on the Co content.

Evolution of transmission Mössbauer spectra of $\mathrm{Fe}_{50} \mathrm{Co}_{50}$ powders with the milling time is presented in Fig. 1. At first glance, the spectra seem to be very similar. They are composed of a dominant (over 98\%), somewhat asymmetric sextet with average hyperfine magnetic field (HMF) about $34.8 \mathrm{~T}$, as well as a small component in the form of low-field sextet. Individual lines in the main sextet are clearly broader in relation to pure $\alpha$-iron. The half width at half maximum (HWHM) is about $0.4-0.5 \mathrm{~mm} / \mathrm{s}$. It is related both to the chemical

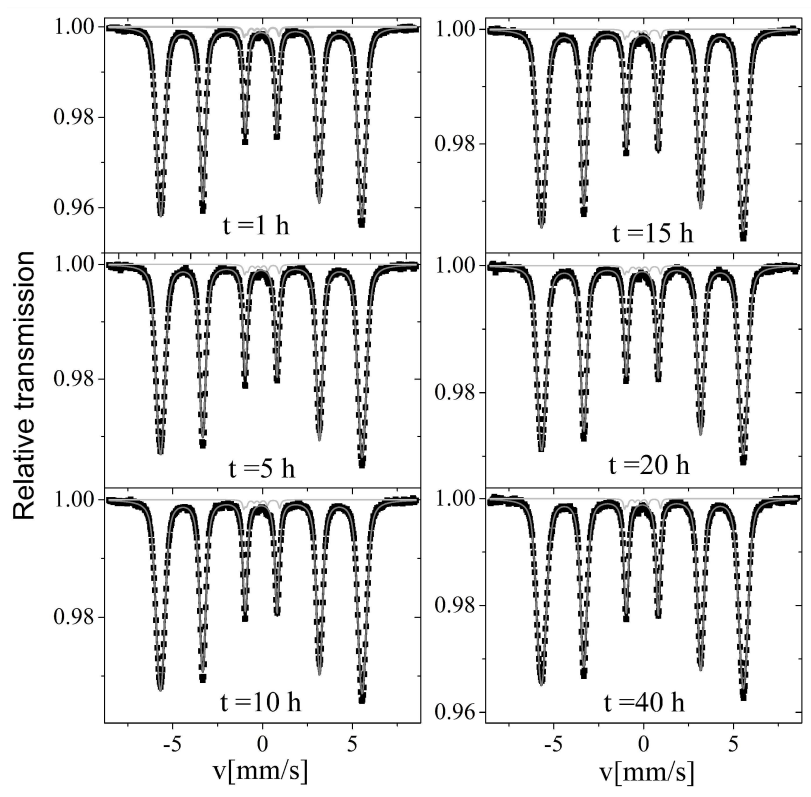

Fig. 1. Evolution of Mössbauer spectra of $\mathrm{Fe}_{50} \mathrm{Co}_{50}$ powders with the milling time $t$.
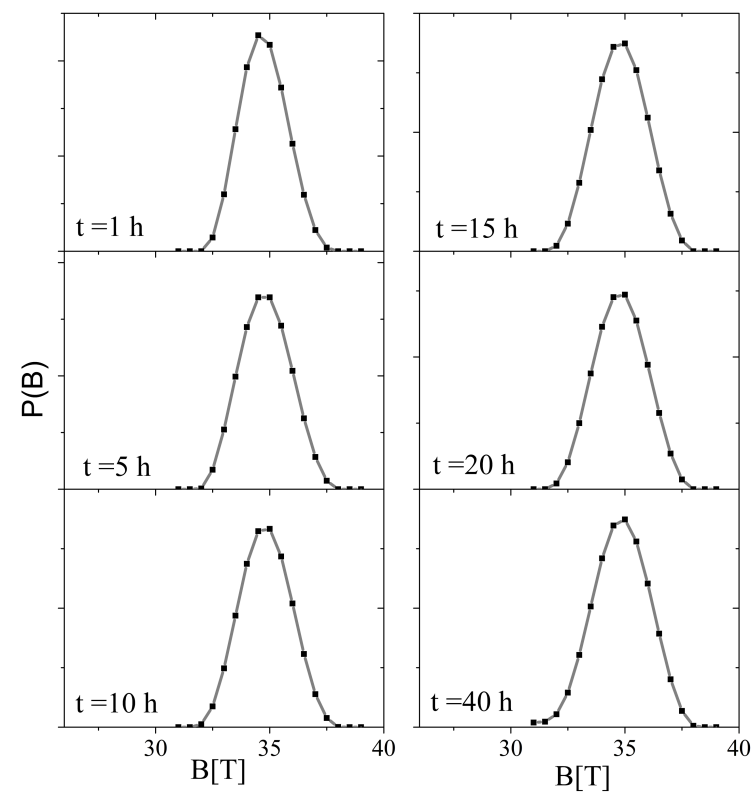

Fig. 2. HMF distributions derived from Mössbauer spectra milled for different time $t$.

and topological disorder in the crystal lattice, manifested by the existence of many inequivalent positions of iron atoms with slightly different values of hyperfine parameters. Therefore, the spectra were fitted by a hyperfine magnetic field distribution, taking into account correlations between local isomer shift and hyperfine magnetic field. After initial numerical analysis the common range of HMF for all the spectra: $31 \mathrm{~T} \leq B \leq 39 \mathrm{~T}$ was chosen in the fitting procedure. Constant relation between line intensities within the sextet $I_{1,6}: I_{2,5}: I_{3,4}=3: 2: 1$ was assumed, as preliminary fitting gave $I_{2,5}$ close to 2 . Even in the powder milled for the shortest time $t=1 \mathrm{~h}$ any component typical of pure iron is not observed in the spectrum, which means that the elemental iron and cobalt powders are mixed at the atomic level.

Figure 2 shows the hyperfine magnetic field distributions $P(B)$, determined as a result of fitting the spectra. All the curves have rather symmetric shape but slightly differ in their positions and widths. The following parameters obtained on the basis of the fitting procedure have been analyzed. These are the average value, skewness and standard deviation of hyperfine magnetic field distribution, and average isomer shift.

As is shown in Fig. 3, the value of average HMF belongs to the range between $34.7 \mathrm{~T}$ after $1 \mathrm{~h}$ of milling and $34.9 \mathrm{~T}$ and small differences are within the error limit. The value of the HMF is close to that reported for mechanically alloyed FeCo which are within the range of $34.8 \mathrm{~T}$ to $35.6 \mathrm{~T}[7-9,13]$.

Skewness (or skew), which is a measure of the asymmetry of the probability distribution of a real-valued random variable about its average value, is positive for distributions obtained for samples milled within $20 \mathrm{~h}$ or less, decreases with the milling time and is clearly negative 


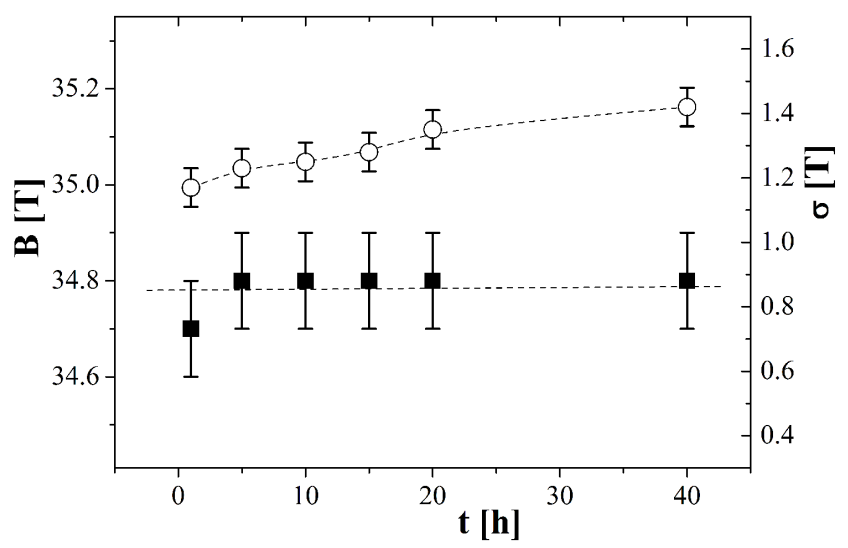

Fig. 3. Average HMF (squares) and standard deviation of HMF (circles) vs. the milling time $t$.

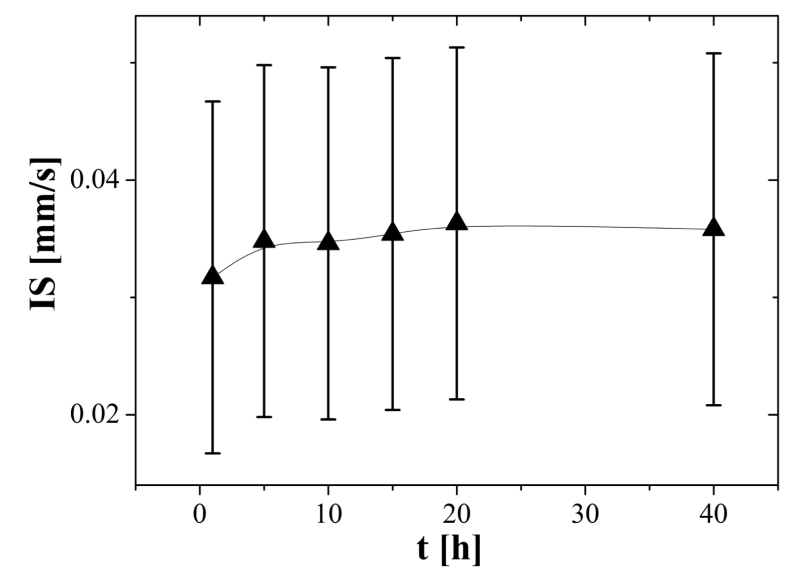

Fig. 4. Average isomer shift vs. the milling time $t$.

for sample milled within $40 \mathrm{~h}$. It means that the HMF distributions derived for powders milled up to $20 \mathrm{~h}$ have a small asymmetry with a tail on the right side while for $t=40 \mathrm{~h}$ the distribution has a tail on the left, lowfield side. This evolution reflects real changes in powder structure with the milling time, towards a greater disorder with a growing number of defects of the crystalline lattice.

In the case of ideally random arrangement of atoms in binary alloys the occupation probability of individual positions in the first and second coordination shell is described by the binomial distribution. Many studies $[17,18]$ analyzed the HMF distribution in iron alloys in terms of the local environment model, introduced by Wertheim et al. [19], which assumes additivity of HMF contributions originated from both iron and solute atoms that surround a chosen Mössbauer nuclei. Within such a model one can determine the actual distribution of occupations and, by comparison with the theoretical distribution, draw conclusions about the actual structure of the alloy. However, an attempt to use a discrete HMF distribution based on the local environment model in order to fit the spectra of mechanically alloyed $\mathrm{Fe}_{50} \mathrm{Co}_{50}$ failed with all samples. This confirms the observations reported earlier [20] that this model is inappropriate for non-dilute $\mathrm{Fe}-\mathrm{Co}$ alloys.

The analysis of standard deviation of HMF (see Fig. 3) showed that this value grows monotonically throughout the whole milling process. This tendency proves the growing disorder of the atom arrangement or/and the increase in internal stresses in the course of milling.

The isomer shift of the main sextet does not change with the milling time within the experimental uncertainties (Fig. 4). The values derived from the Mössbauer spectra are close to those obtained by Moumeni et al. [9] for mechanically alloyed $\mathrm{Fe}-\mathrm{Co}$ powders and are slightly larger than the results obtained for bulk disordered $\mathrm{Fe}-$ Co alloys [20] (about $0.037 \mathrm{~mm} / \mathrm{s}$ at $77 \mathrm{~K}$ ). Therefore, the discrepancy can be related to the mechanical alloying process.

In addition to the main sextet, also a small low-field component in the spectra is present, the relative intensity of which does not exceed $1.7 \%$ and the value of $\mathrm{HMF}$ is close to/or less than $6 \mathrm{~T}$. This component can be related to trace phases containing impurities coming from the mill. It cannot be ruled out that the component is an artefact of the fitting procedure resulting from the assumption of the ratio of line intensities in the sextet although this seems rather unlikely.

\section{Conclusions}

The bcc iron-cobalt solid solution was identified by the Mössbauer spectroscopy. There are no indications of the presence of pure iron in the ground material. Therefore, all of the iron was alloyed with cobalt after just one hour of grinding. Both average isomer shift and average hyperfine magnetic field remained stable in the examined range of the milling time. Structural evolution of the investigated alloys consisting in growing disorder manifested itself through subtle changes in shape of the HMF distribution. The distribution was slightly asymmetric and its tail moved from right side of the maximum to the left one with increasing milling time. A regular rise in the standard deviation is observed, indicating an increasing topological disorder and/or increase in internal strains.

From a technological point of view, it is worth emphasizing that it is possible to choose the optimal, relatively short milling time when the $\mathrm{Fe}_{50} \mathrm{Co}_{50}$ alloy is already formed and at the same time the number of defects does not increase significantly due to long grinding.

\section{Acknowledgments}

This work was supported by the Scientific Grant Agency of the Ministry of Education of the Slovak Republic and the Slovak Academy of Sciences KEGA 002TUKE-4/2019 and VEGA 1/0301/20. 


\section{References}

[1] C. Suryanarayana, Prog. Mater. Sci. 46, 1 (2001).

[2] J. Ding, Y. Shi, L.F. Chen, C.R. Deng, S.H. Fuh, Y. Li, J. Magn. Magn. Mater. 247, 249 (2002).

[3] H. Gleiter, Nanostruct. Mater. 6, 3 (1995).

[4] C. Suryanarayana, C.C. Koch, Hyperfine Interact. 130, 5 (2000).

[5] H.W. Song, S.R. Guo, Z.Q. Hu, Nanostruct. Mater. 11, 203 (1999).

[6] Ch. Kuhrt, L. Schultz, J. Appl. Phys. 71, 1896 (1992).

[7] R. Brüning, K. Samwer, C. Kuhrt, L. Schultz, J. Appl. Phys. 72, 2978 (1992).

[8] M. Sorescu, A. Grabias, Intermetallics 10, 317 (2002).

[9] H. Moumeni, S. Alleg, J.M. Grenèche, J. Alloys Comp. 386, 12 (2005).

[10] P. Rajesh, J.-M. Greneche, G.A. Jacob, T. Arun R.J. Joseyphus, Phys. Status Solidi A 216, 1900051 (2019).
[11] V.A. Bautin, S.A. Gudoshnikov, A.G. Seferyan, N.A. Usov, J. Supercond. Nov. Magn. 30, 1281 (2017).

[12] T. Sourmail, Prog. Mater. Sci. 50, 816 (2005).

[13] A. Zeleňáková, D. Olekšáková, J. Degmová, J. Kováč, P. Kollár, M. Kusý, P. Sovák, J. Magn. Magn. Mater. 316, e519 (2007).

[14] D. Olekšáková, P. Kollár, J. Füzer, F. Onderko, S. Dobák, J. Viňáš, M. Fáberová, R. Bureš, Acta Phys. Pol. A 131, 807 (2017).

[15] C. Kuhrt, L. Schultz, J. Appl. Phys. 73, 6588 (1993).

[16] J. Hesse, A. Rübartsch, J. Phys. E Sci. Instrum. 7, 526 (1974).

[17] E. Jartych, J. Magn. Magn. Mater. 265, 176 (2003).

[18] R. Idczak, R. Konieczny, J. Chojcan, Nukleonika 60. 39 (2015)

[19] G.K. Wertheim, V. Jaccarino, J.H. Wernick, D.N.E. Buchanan, Phys. Rev. Lett. 12, 24 (1964).

[20] H.H. Hamdeh, B. Fultz, D.H. Pearson, Phys. Rev. B 39, 11233 (1989). 